\title{
Estudio piloto sobre entrenamiento físico durante hemodiálisis
}

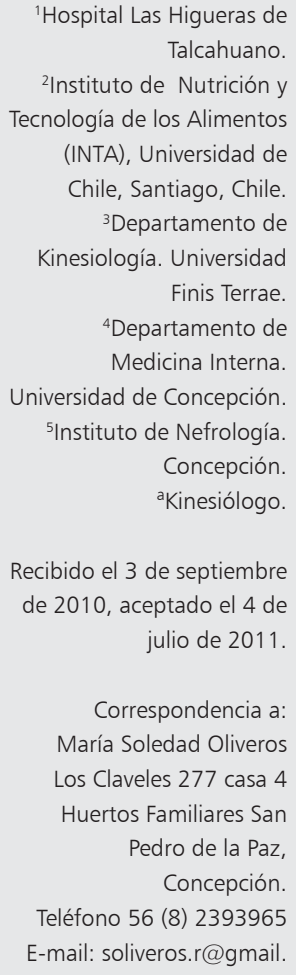

${ }^{1}$ Hospital Las Higueras de Talcahuano.

${ }^{2}$ Instituto de Nutrición y Tecnología de los Alimentos (INTA), Universidad de Chile, Santiago, Chile.

${ }^{3}$ Departamento de Kinesiología. Universidad Finis Terrae.

${ }^{4}$ Departamento de Medicina Interna. Universidad de Concepción. Instituto de Nefrología. Concepción. ${ }^{a}$ Kinesiólogo.

Recibido el 3 de septiembre de 2010, aceptado el 4 de julio de 2011.

Correspondencia a: María Soledad Oliveros Los Claveles 277 casa 4 Huertos Familiares San Pedro de la Paz, Concepción. Teléfono 56 (8) 2393965 E-mail: soliveros.r@gmail.

\section{A pilot study on physical training of patients in hemodialysis}

Background: Exercise training during the dialytical procedure may have positive cardiovascular effects and prevent or revert muscle wasting in patients undergoing chronic hemodialysis. Aim: To evaluate the effects of an exercise training program in patients undergoing chronic hemodialysis. Material and Methods: Fifteen patients on chronic hemodialysis aged 21 to 69 years (three females) were included in the study. Nine of these were included in an exercise training program. During 16 weeks, exercise sessions were carried out during each dialytical procedure that included a warm-up period, aerobic exercises done using standing cycles, and resistance exercises, performed using Thera-Band ${ }^{\circledast}$ elastic bands and loops. Borg scale was used to control the intensity of training. At baseline and at the end of the study, a blood sample prior and after the dialytical procedure was obtained to measure $C$ reactive protein, tumor necrosis factor $\alpha$ and interleukin 6. Quadriceps muscle strength, six minutes' walk and quality of life using the SF-36 questionnaire, were also measured. Results: Four experimental subjects did not complete the study period, two that withdrew before starting, one due to problems with the venous access and one that decided to withdraw after 1 month of training. Among the five patients that finished the training period, significant improvements in the six minutes' walk and quadriceps strength were observed in the experimental group. No significant changes were observed among controls. No changes were observed in either group in C reactive protein, tumor necrosis factor and interleukin 6 levels or quality of life. Conclusions: Among patients undergoing chronic hemodialysis exercise training improves endurance and muscle strength.

(Rev Med Chile 2011; 139: 1046-1053).

Key words: Muscle, skeletal; Renal dialysis; Resistance training.
M ás de $50 \%$ de los pacientes con enfermedad renal crónica (ERC) terminal tratados con hemodiálisis (HD) crónica mueren de enfermedades cardiovasculares ${ }^{1}$. Un grupo de alteraciones presentes en los pacientes con ERC terminal, son las alteraciones musculares, siendo éstas los factores limitantes más importantes de su capacidad funcional ${ }^{2}$. El estilo de vida sedentario es una de las principales causas de la baja capacidad física y un factor de riesgo independiente de muerte en pacientes con ERC terminal sometidos a $\mathrm{HD}^{3}$. La capa- cidad física de los pacientes con ERC terminal corresponde al $60 \%$ de una población de la misma edad con función renal normal ${ }^{4}$. Además hay una declinación progresiva de la capacidad funcional después del inicio de programas de hemodiálisis crónica ${ }^{5}$.

La terapia de reemplazo renal puede atenuar algunas de las anormalidades metabólicas de la ERC, pero la pérdida de aminoácidos durante procedimientos dialíticos y la liberación de citoquinas, llevan a un mayor catabolismo proteico muscular y síntesis de proteínas de fase aguda ${ }^{6}$. Los niveles 
elevados de citoquinas son además predictores de mortalidad cardiovascular ${ }^{7}$.

Programas que combinan ejercicio aeróbico y de resistencia pueden aumentar la fuerza y resistencia muscular, la capacidad funcional y la calidad de vida de pacientes en hemodiálisis ${ }^{8}$. El ejercicio aeróbico y de resistencia muscular durante la diálisis aumentan el flujo sanguíneo a nivel muscular con un aumento del área de superficie capilar, lo que dinamiza el flujo de urea y toxinas desde los tejidos hacia el compartimiento vascular, lo que pudiera mejorar la eficacia de la diálisis9. Además el ejercicio regular moderado puede modular la respuesta inflamatoria, lo que sería beneficioso en ERC terminal ${ }^{10}$.

Por lo tanto, el objetivo de este estudio fue evaluar los posibles efectos funcionales y sobre los niveles de citoquinas, de un programa de ejercicio aeróbico y de resistencia durante la hemodiálisis en pacientes con ERC.

\section{Material y Método}

\section{Pacientes}

Fueron reclutados 15 pacientes, pertenecientes a cuatro turnos de HD, en tratamiento permanente de HD, de una población de 123 pacientes, perteneciente a un Centro de Diálisis de la comuna de Pedro Aguirre Cerda, en la zona sur de Santiago. Los pacientes fueron incluidos si se encontraban en HD por más de 3 meses, tenían un hematocrito mayor de 30\%, una ganancia de peso interdiálisis menor a 1 kilo por día y un potasio prediálisis menor a 5,5 mmol/L. Los criterios de exclusión incluyeron: diagnóstico de infarto miocárdico en las 6 semanas previas, angina inestable, amputación de miembros inferiores, presencia de alguna alteración neurológica con déficit funcional, alteraciones musculo esqueléticas o respiratorias que empeoren con el ejercicio, y diabetes mellitus descompensada.

El Comité de Ética del Instituto de Nutrición y Tecnología de los Alimentos (INTA), de la Universidad de Chile, aprobó el estudio, así como también el Director Médico del Centro de Diálisis en cuestión, y además todos los pacientes del estudio firmaron un consentimiento informado.

\section{Diseño del estudio}

Los pacientes estudiados pertenecen a cuatro turnos de HD, en el mismo día consecutivo de la semana (lunes-miércoles-viernes; primero, segundo, tercero y cuarto turno). Se dividieron en un grupo experimental, quienes realizaron el programa de ejercicio, y en un grupo control, tratando de parearlos por edad y antecedentes clínicos. Todos los pacientes realizaron, al inicio y al final del estudio, un test de marcha de 6 minutos (6MWT), evaluación de la fuerza muscular de cuádriceps, encuestas de calidad de vida y de depresión, y medición de parámetros inflamatorios séricos, proteína $\mathrm{C}$ reactiva ultrasensible (PCRus), factor de necrosis tumoral $\alpha(\mathrm{TNF} \alpha)$ e interleukina 6 (IL-6). Estos últimos parámetros se midieron antes e inmediatamente después de la sesión de diálisis en cada período de estudio.

Los pacientes del grupo experimental completaron 16 semanas de ejercicio programado durante la sesión de hemodiálisis, de acuerdo al siguiente protocolo:

\section{Protocolo de ejercicio}

Los pacientes se ejercitaron bajo la supervisión de un kinesiólogo y médico a cargo, con una frecuencia de 3 veces por semana, realizada durante las 2 primeras horas de la HD. Se efectuaron ejercicios aeróbicos y de resistencia muscular. Cada sesión duraba 45 a 60 minutos, la cual incluyó una etapa de calentamiento, etapa de ejercicio y etapa de vuelta a la calma ${ }^{11}$.

\section{Intervención de ejercicio aeróbico}

El ejercicio aeróbico consistió en la realización de ejercicios continuos por un tiempo de 20 a 25 minutos, que involucraran los grupos musculares de la extremidades inferiores, a través de una pedalera. La intensidad de trabajo se controló a través de la frecuencia cardiaca (FC) con relojes POLAR ${ }^{\circledR}$, y la estimación de la intensidad de trabajo se determinó por la frecuencia cardiaca de reserva (FCr). Se trabajó a intensidades moderadas, que van de $40-60 \%$ de la FCr. Esta corresponde a $[(220$-edad $)$-frecuencia cardiaca de reposo $)]^{*}(40$ a $60 \%)+$ frecuencia cardiaca de reposo ${ }^{12}$. Se utilizó además la escala de Borg 13 que mide el cansancio subjetivo, para moderar la carga de ejercicio, a la que se dio más importancia en pacientes que usaban beta bloqueadores.

Intervención de ejercicio de resistencia muscular

El programa de ejercicio consistió en realizar ejercicios isotónicos de resistencia progresiva 
usando bandas elásticas Thera-Band ${ }^{\circledR}$, con el objetivo de potenciar los miembros inferiores, realizando un ejercicio adaptado a la posición del paciente durante la sesión de HD. Cada sesión de ejercicio de resistencia muscular tuvo una duración de entre 20 a 25 minutos. El protocolo de progresión de ejercicio de resistencia se estableció mediante la relación entre el desempeño motor del paciente y la percepción de su esfuerzo.

Se realizaron ejercicios de extensión de rodilla uni y bilateral. La banda elástica Thera-Band ${ }^{\circledR}$ se ubicó en el extremo distal del segmento a movilizar, permitiendo el movimiento completo de la articulación involucrada en el plano óptimo; se supervisó que los segmentos proximales se mantuvieran estabilizados, con el objetivo de evitar sobrecargas innecesarias en estructuras o tejidos blandos adyacentes.

Se detuvo el protocolo de ejercicio si durante éste el paciente presentaba una presión arterial mayor de 180/110 o menor de 110/50 o una caída brusca de ésta, estuviera muy cansado para mantener el nivel de exigencia, opresión precordial, disnea, náuseas, palpitaciones, calambres, sensación de mareo, y dolor muscular o articular ${ }^{14}$.

\section{Parámetros clínicos de los pacientes}

En el período basal y al final del estudio, a todos los pacientes se les midió nitrógeno ureico pre y post diálisis, con lo que se calculó el Kt/v (Valor Mínimo aceptado por "Kidney Disease Outcomes Quality Initiative" (KDOQI): 1,2). Además se midieron hematocrito, calcio, fósforo, creatininemia y potasio sérico.

\section{Test de marcha de 6 minutos (6MWT)}

El test de marcha de 6 minutos se realizó al inicio y al final del programa de ejercicio, tanto en el grupo control como en el grupo experimental; fue realizado de acuerdo con el protocolo recomendado por la Sociedad Americana del Tórax ${ }^{15}$. Este test fue realizado por el kinesiólogo y médico a cargo del estudio.

\section{Fuerza muscular}

Se midió fuerza muscular de cuádriceps con un equipo manual que expresa la fuerza máxima expresada en kilogramos (Nicolas Manual Muscle TesterLafayette Instruments). Este procedimiento se repite 3 veces en forma alternada para cada extremidad.

\section{Test de calidad de vida}

El cuestionario "Medical Outcomes Survey short form (SF-36)" se utilizó para evaluar la calidad de vida. La versión chilena fue traducida y validada por Albala et al. Sus 36 ítems dan información sobre 8 subescalas, puntuándose cada una de 0 (peor nivel de salud) a 100 (mejor nivel de salud) ${ }^{16}$. Los pacientes completaron el cuestionario de forma independiente, con la ayuda de los investigadores cuando fue necesario.

\section{Test de depresión de Beck (BDI-I)}

El test de Beck evalúa en forma cuantitativa la intensidad de las manifestaciones conductuales de la depresión (BDI), y es capaz de evaluar los cambios de ésta en un intervalo de tiempo. El test está constituido por 21 ítem. Se interpreta la puntuación total de la escala (5-9: altibajos normales; 10-19: depresión leve-moderada; 20-29: depresión moderada-severa; 29-63: depresión severa) ${ }^{17}$. Los pacientes completaron el cuestionario de forma independiente, con la ayuda de los investigadores cuando fue necesario. La versión española de este test está validada.

\section{Medición PCRus, TNF- $\alpha, I L-6$}

Para la medición de PCR se realizó un ELISA en fase sólida, utilizando un kit comercial PCR ultrasensible (DRG Internacional Inc, USA). Para la medición de TNFo e IL-6 se realizó un ensayo inmunométrico quimioluminiscente, automatizado para IMMULITE 1000 (IMMULITE ${ }^{\circledR}$ TNF $\alpha$, e IMMULITE $^{\circledR}$ IL6 de SIEMENS Healthcare Diagnostics Products Ltd). Todos los ensayos se hicieron en una ocasión, al final del estudio. Las muestras fueron guardadas a $-80^{\circ} \mathrm{C}$ hasta el momento del ensayo

\section{Análisis de los datos}

La normalidad de la distribución de las variables se evaluó con la prueba de Shapiro-Wilk. Las variables con una distribución normal, se expresaron como promedio \pm desviación estándar. Las variables sin distribución normal se expresaron como mediana y rangos. Se efectuó un análisis por protocolo en que sólo se incluyeron aquellos pacientes que terminaron el período de observación, excluyendo a los que desertaron. Los cambios de variables paramétricas se evaluaron utilizando la prueba de t pareado. Para evaluar diferencias entre las deltas, entre el grupo control y experimental, 
al inicio y al final del programa de ejercicio, se utilizó prueba t de Student para variables con distribución normal y la prueba de Wilcoxon para variables sin distribución normal. Se tomó como significativo un valor de $\mathrm{p}<0,05$.

\section{Resultados}

Las características clínicas de los pacientes están resumidas en la Tabla 1. Quince pacientes en HD crónica fueron reclutados desde el Centro de Diálisis Nephrocare, de la comuna de Pedro Aguirre Cerda. De éstos, nueve se asignaron al grupo experimental y seis al grupo control. En el grupo experimental, dos pacientes desertaron durante la primera semana de estudio, por lo que sólo realizaron las pruebas iniciales; un paciente tuvo que retirarse del estudio a la tercera semana por dificultad en su acceso venoso, que le impedía realizar correctamente el programa de ejercicios; un paciente desertó del estudio a la cuarta semana, por lo que alcanzó a realizar las pruebas iniciales más un mes de ejercicio. Respecto al grupo control, no hubo deserciones. El ejercicio sólo se detuvo, de acuerdo a los criterios de detención, en el paciente con problemas en el acceso venoso, señalado anteriormente.

Las Tablas 2 y 3 muestran la evolución del test de 6 minutos y fuerza de cuádriceps en los pacientes estudiados, respectivamente. En los

Tabla 1. Características clínicas de los pacientes en diálisis

\begin{tabular}{|lccc|}
\hline Variable & Grupo control & Grupo experimental & p \\
\hline Edad & $52,2 \pm 17^{\S}$ & $46 \pm 13$ & NS \\
\hline No mujeres $^{*}$ & $3^{\pi}$ & 0 & NS \\
\hline No hombres $^{*}$ & 3 & 5 & NS \\
Peso seco & $73,3 \pm 9,3$ & $74,5 \pm 5,5$ & NS \\
\hline Peso post-hemodiálisis & $73,8 \pm 9$ & $74,5 \pm 6$ & NS \\
Diabetes Mellitus & 0 & 1 & NS \\
\hline Hipertensión arterial & 6 & 4 & \\
\hline
\end{tabular}

$\S=$ promedio \pm desviación estándar. " = número de observaciones.

Tabla 2. Distancia recorrida en el 6MWT (metros, promedio \pm desviación estándar)

\begin{tabular}{|lcccccc|}
\hline & Inicio & Final & t pareado & Delta & $\begin{array}{c}\text { t delta control-delta } \\
\text { experimental }\end{array}$ \\
\hline Control & $(n=6)$ & $512 \pm 93$ & $506 \pm 75$ & $p=0,72$ & $-5,6$ & \\
Experimental & $(n=5)$ & $596 \pm 79$ & $630 \pm 85$ & $p=0,05$ & 34,4 & $p=0,037$ \\
\hline
\end{tabular}

Tabla 3. Fuerza muscular de cuádriceps (kg, promedio \pm desviación estándar)

\begin{tabular}{|c|c|c|c|c|c|c|}
\hline & & Inicio & Final & t pareado & Delta & $\begin{array}{l}\text { t delta control-delta } \\
\text { experimental }\end{array}$ \\
\hline \multicolumn{7}{|c|}{ Pierna derecha } \\
\hline Control & $(n=6)$ & $17,9 \pm 4,2$ & $18,9 \pm 4,6$ & $p=0,27$ & 0,93 & $p=0,027$ \\
\hline Experimental & $(n=5)$ & $15,4 \pm 5$ & $19,3 \pm 3,9$ & $p=0,03$ & 3,86 & \\
\hline \multicolumn{7}{|c|}{ Pierna izquierda } \\
\hline Control & $(n=6)$ & $17,4 \pm 3,3$ & $19,4 \pm 4,4$ & $p=0,06$ & 2,03 & $p=0,054$ \\
\hline Experimental & $(n=5)$ & $18 \pm 4,4$ & $21 \pm 4$ & $p=0,02$ & 3 & \\
\hline
\end{tabular}


Tabla 4. Marcadores inflamatorios séricos antes e inmediatamente después de la sesión de diálisis en el período basal y después de 16 semanas, expresados como mediana (rango)

\begin{tabular}{|c|c|c|c|}
\hline & Grupo control $(n=6)$ & Grupo experimental $(n=5)$ & $\mathbf{p}$ \\
\hline \multicolumn{4}{|c|}{ Proteína $\mathrm{C}$ reactiva $(\mathrm{mg} / \mathrm{L})$} \\
\hline Prediálisis basal & $7,55(5,7-100)$ & $6,2(5,3-12,3)$ & NS \\
\hline Postdiálisis basal & $8,1 \quad(5,7-100)$ & $6,8(5,4-10,3)$ & NS \\
\hline Prediálisis final & $5,4 \quad(4,9-63,4)$ & $6,2(5,4-7,4)$ & NS \\
\hline Postdiálisis final & $7,85(5,3-85,7)$ & $6,1(5,1-7,5)$ & NS \\
\hline \multicolumn{4}{|c|}{ Factor de necrosis tumoral $\alpha(\mathrm{pg} / \mathrm{ml})$} \\
\hline Prediálisis basal & $27 \quad(19,4-31,5)$ & $(17-22,2)$ & $=0,02$ \\
\hline Postdiálisis basal & $27,25(20,1-33,8)$ & $25,9 \quad(17,2-48,5)$ & NS \\
\hline Prediálisis final & $27,95(24,6-56,9)$ & $18,7 \quad(14,8-29,7)$ & NS \\
\hline Postdiálisis final & $28,15(20,7-32,2)$ & $24,3 \quad(15,6-32,7)$ & NS \\
\hline \multicolumn{4}{|l|}{ Interleukina 6 (pg/ml) } \\
\hline Prediálisis basal & $6,78(3,53-15,1)$ & $3,77(2,76-22,6)$ & NS \\
\hline Postdiálisis basal & $6,3(4,8-14,8)$ & $5,87(4,17-7,39)$ & NS \\
\hline Prediálisis final & $7,88(2,25-19,2)$ & $4,95(2,51-5,04)$ & NS \\
\hline Postdiálisis final & $6,68(2,89-13,5)$ & $5,86(2,57-8,57)$ & NS \\
\hline
\end{tabular}

pacientes experimentales, la capacidad de marcha aumentó significativamente al final del período de entrenamiento y no se observaron cambios significativos en el grupo control. La fuerza de cuádriceps derecho aumentó significativamente en los pacientes experimentales. La fuerza de cuádriceps izquierdo también aumentó más en los pacientes experimentales, pero la diferencia no alcanzó significación estadística

Los marcadores inflamatorios evaluados PCR, TNF $\alpha$ e IL6, en el grupo control y experimental, tanto al inicio como al final del programa de ejercicio se muestran en la Tabla 4. No encontramos ninguna diferencia significativa tras la realización de la intervención.

$\mathrm{El} \mathrm{Kt} / \mathrm{v}$ medido al inicio en el grupo control fue de 1,4 $\pm 0,04$ y en el grupo experimental de $1,2 \pm 0,02$; tras las 16 semanas de ejercicio, el grupo control tuvo un Kt/v promedio de 1,5 \pm 0,07 y el grupo experimental 1,2 $\pm 0,08$, sin observarse una diferencia significativa tras la realización del programa de ejercicio.

No observamos cambios en los puntajes de calidad de vida o depresión en ningún grupo (Tabla 5).

\section{Discusión}

El programa de ejercicio intradiálisis resultó en un mejoramiento del rendimiento físico. La distancia recorrida en el test de 6 minutos aumentó $5,7 \%$ en el grupo experimental, en respuesta al programa de ejercicios de 16 semanas, lo que indica una mejora significativa en la función física en los sujetos que se ejercitaron.

Nuestros resultados en el 6MWT son semejantes a los obtenidos por Ridley et al $^{18}$, donde se observó un incremento de $14 \%$ en la distancia recorrida en el 6MWT, en 18 pacientes en $\mathrm{HD}$, que realizaron un programa de ejercicio de 12 semanas con ejercicio aeróbico. Painter et al ${ }^{19}$ encontraron un aumento del $8 \%$ en la distancia recorrida en 6MWT en 44 pacientes que participaron en un programa domiciliario de 8 semanas y ejercicio intradiálisis por 8 semanas.

La fuerza muscular en la extremidad inferior derecha aumentó en 5,5\% en el grupo control, y $25 \%$ en el experimental $(p=0,03)$, en respuesta al programa de ejercicio; $y$ en la extremidad inferior izquierda aumentó $11,5 \%$ y $17 \%$ (NS), respectivamente, lo que coincide y probablemente se asocia con el aumento de la capacidad de marcha. 
Tabla 5. Puntaje de la encuesta de calidad de vida relacionada a la salud (SF-36) y de la escala de depresión de Beck (promedio \pm desviación estándar)

\begin{tabular}{|c|c|c|c|c|}
\hline & & $\begin{array}{l}\text { Pacientes controles } \\
(n=6)\end{array}$ & $\begin{array}{l}\text { Pacientes experimentales } \\
\qquad(n=5)\end{array}$ & $\mathbf{p}$ \\
\hline $\begin{array}{l}\text { Subescalas del SF-3 } \\
\text { Funcionamiento físico }\end{array}$ & $\begin{array}{l}6 \\
\text { basal } \\
16 \text { semanas }\end{array}$ & $\begin{array}{l}60,8 \pm 32,3 \\
60,8 \pm 31,2\end{array}$ & $\begin{array}{l}82,0 \pm 15,2 \\
75,0 \pm 24,7\end{array}$ & $\begin{array}{l}\text { NS } \\
\text { NS }\end{array}$ \\
\hline Rol físico & $\begin{array}{l}\text { basal } \\
16 \text { semanas }\end{array}$ & $\begin{array}{l}41,7 \pm 25,8 \\
79,2 \pm 24,6\end{array}$ & $\begin{array}{l}40,0 \pm 33,5 \\
45,0 \pm 51,2\end{array}$ & $\begin{array}{l}\text { NS } \\
\text { NS }\end{array}$ \\
\hline Dolor corporal & $\begin{array}{l}\text { basal } \\
16 \text { semanas }\end{array}$ & $\begin{array}{l}77,2 \pm 14,2 \\
77,8 \pm 14,0\end{array}$ & $\begin{array}{l}75,0 \pm 20,0 \\
71,1 \pm 21,7\end{array}$ & $\begin{array}{l}\text { NS } \\
\text { NS }\end{array}$ \\
\hline Salud mental & $\begin{array}{l}\text { basal } \\
16 \text { semanas }\end{array}$ & $\begin{array}{l}74,0 \pm 18,0 \\
66,7 \pm 15,3\end{array}$ & $\begin{array}{l}85,6 \pm 9,2 \\
81,6 \pm 10,0\end{array}$ & $\begin{array}{l}\text { NS } \\
\text { NS }\end{array}$ \\
\hline Rol emocional & $\begin{array}{l}\text { basal } \\
16 \text { semanas }\end{array}$ & $\begin{array}{l}61,0 \pm 49,1 \\
66,7 \pm 29,8\end{array}$ & $\begin{array}{l}93,2 \pm 15,2 \\
80,0 \pm 44,7\end{array}$ & $\begin{array}{l}\text { NS } \\
\text { NS }\end{array}$ \\
\hline Funcionamiento social & $\begin{array}{l}\text { basal } \\
16 \text { semanas }\end{array}$ & $\begin{array}{l}75,3 \pm 19,2 \\
77,8 \pm 21,1\end{array}$ & $\begin{array}{l}86,0 \pm 9,6 \\
86,7 \pm 9,3\end{array}$ & $\begin{array}{l}\text { NS } \\
\text { NS }\end{array}$ \\
\hline Vitalidad & $\begin{array}{l}\text { basal } \\
16 \text { semanas }\end{array}$ & $\begin{array}{l}67,5 \pm 11,3 \\
61,7 \pm 18,3\end{array}$ & $\begin{array}{l}81,0 \pm 13,9 \\
78,0 \pm 13,5\end{array}$ & $\begin{array}{l}\text { NS } \\
\text { NS }\end{array}$ \\
\hline Salud general & $\begin{array}{l}\text { basal } \\
16 \text { semanas }\end{array}$ & $\begin{array}{l}57,2 \pm 18,2 \\
60,5 \pm 20,3\end{array}$ & $\begin{array}{l}57,2 \pm 19,7 \\
64,2 \pm 17,7\end{array}$ & $\begin{array}{l}\text { NS } \\
\text { NS }\end{array}$ \\
\hline Puntaje de depresió & $\begin{array}{l}\text { ón de Beck } \\
\text { basal } \\
16 \text { semanas }\end{array}$ & $\begin{array}{l}11,3 \pm 2,5 \\
14,7 \pm 7,3\end{array}$ & $\begin{array}{l}10,6 \pm 4,9 \\
13,4 \pm 8,0\end{array}$ & $\begin{array}{l}\text { NS } \\
\text { NS }\end{array}$ \\
\hline
\end{tabular}

La mejoría observada en la capacidad funcional tras el programa de ejercicio, no se asoció con un cambio en el nivel de depresión, evaluada con el test de Beck. Tampoco hubo una mejoría en la percepción de la calidad de vida, evaluada con el test SF-36. Estudios previos tampoco encontraron ningún cambio en la percepción de la calidad de vida, evaluada con SF-36, tras un programa de entrenamiento de 8 semanas intradiálisis en 6 pacientes ${ }^{20}$. Sin embargo, OhPark et $\mathrm{al}^{21}$ encontraron una mejoría significativa en el componente mental y componente físico del test SF-36, en 18 pacientes en HD después de un programa de entrenamiento de 3 meses de ejercicio intradiálisis.

Los parámetros inflamatorios y el Kt/v eva- luados en los pacientes del grupo control y experimental no variaron significativamente tras la realización del programa de ejercicio. En la literatura no encontramos un estudio que evaluara los parámetros inflamatorios en cuestión, al realizar un programa de ejercicio en pacientes en $\mathrm{HD}$, realizado intradiálisis. Viana et al, realizaron ejercicio aeróbico a 16 pacientes con ERC etapa IV y V pre-diálisis, observando una disminución significativa en la concentración plasmática de IL-6, pero no de PCR (Congreso Mundial de Nefrología. Milán 2009). Nuestra hipótesis estaba basada en que al mejorar y aumentar la depuración de desechos y toxinas urémicas, desde el compartimento muscular, esto se vería reflejado en una disminución de los parámetros inflama- 
torios y en la eficiencia de la diálisis. El pequeño número de pacientes estudiados no nos permite descartar un error tipo 2 en la evaluación de los parámetros inflamatorios, pero al ver las medianas de valores, es poco probable que se obtengan diferencias significativas al aumentar la muestra. Es también probable que el diseño del estudio no permita determinar cambios en estos marcadores.

Este estudio tiene tres debilidades que deben tenerse en cuenta. La muestra estudiada es pequeña y puede disminuir el poder para encontrar efectos del entrenamiento físico. El motivo de escoger esta muestra pequeña fue que este estudio se diseñó como un piloto para ver la factibilidad de efectuar entrenamiento físico a pacientes en diálisis. La segunda debilidad es que los pacientes no se seleccionaron al azar para incluirse en el grupo control o experimental, sino que se parearon para que controles y experimentales tuvieran características similares. Este tipo de asignación puede provocar problemas pero es indispensable cuando se trabaja con grupos pequeños. La tercera debilidad es que tanto los parámetros inflamatorios como el Kt/v sólo se midieron al inicio y al final del estudio y no con intervalos regulares durante éste. El motivo de esto es el costo implicado, pero mediciones más frecuentes pueden ayudar a detectar diferencias más sutiles.

En conclusión, este estudio muestra que un programa combinado de ejercicios aeróbicos y de resistencia muscular, realizado a pacientes durante la $\mathrm{HD}$, puede llevarse a cabo de forma segura, con una supervisión adecuada; y mejora la capacidad funcional y física de los pacientes con enfermedad renal terminal. Bien diseñado, el entrenamiento físico durante la hemodiálisis puede añadir nuevas áreas a la atención de la población en diálisis.

Agradecimientos: A Thera-Band Academy, quienes proporcionaron los elementos para efectuar todos los ejercicios de entrenamiento de resistencia. Thera-Band Academy no tuvo ingerencia en el diseño de este estudio.

\section{Referencias}

1. Ritz E, Bommer J. Cardiovascular problems on hemodialysis: current deficits and potential improvement. Clin J Am Soc Nephrol 2009; 4 Suppl 1: S71-8.

2. Fahal IH, Bell GM, Bone JM, Edwards RH. Physiological abnormalities of skeletal muscle in dialysis patients.
Nephrol Dial Transplant 1997; 12: 119-27.

3. Carrero JJ, Chmielewski M, Axelsson J, Snaedal S, Heimbürger O, Bárány P, et al. Muscle atrophy, inflammation and clinical outcome in incident and prevalent dialysis patients. Clin Nutr 2008; 27: 557-64.

4. Painter P, Messer-Rehak D, Hanson P, Zimmerman SW, Glass NR. Exercise capacity in hemodialysis, CAPD, and renal transplant patients. Nephron 1986; 42: 47-51.

5. Kurella Tamura M, Covinsky KE, Chertow GM, Yaffe K, Landefeld CS, McCulloch CE. Functional status of elderly adults before and after initiation of dialysis. N Engl J Med 2009; 361: 1539-47.

6. Raj DS, Sun Y, Tzamaloukas AH. Hypercatabolism in dialysis patients. Curr Opin Nephrol Hypertens 2008; 17: 589-94.

7. Barreto DV, Barreto FC, Liabeuf S, Temmar M, Lemke HD, Tribouilloy C, et al. Plasma interleukin-6 is independently associated with mortality in both hemodialysis and pre-dialysis patients with chronic kidney disease. Kidney Int 2010; 77: 550-6.

8. van Vilsteren MC, de Greef MH, Huisman RM. The effects of a low-to-moderate intensity pre-conditioning exercise programme linked with exercise counselling for sedentary haemodialysis patients in The Netherlands: results of a randomized clinical trial. Nephrol Dial Transplant 2005; 20: 141-6.

9. Parsons TL, Toffelmire EB, King-VanVlack CE. Exercise training during hemodialysis improves dialysis efficacy and physical performance. Arch Phys Med Rehabil 2006; 87: 680-7.

10. Beavers KM, Brinkley TE, Nicklas BJ. Effect of exercise training on chronic inflammation. Clin Chim Acta 2010; 411: 785-93.

11. American College of Sports Medicine. American College of Sports Medicine position stand. Progression models in resistance training for healthy adults. Med Sci Sports Exerc 2009; 41: 687-708.

12. Goldberg L, Elliot DL, Kuehl KS. Assessment of exercise intensity formulas by use of ventilatory threshold. Chest 1988; 94 (1): 95-8.

13. Grant S, Aitchison T, Henderson E, Christie J, Zare S, McMurray J, et al. A comparison of the reproducibility and the sensitivity to change of visual analogue scales, Borg scales, and Likert scales in normal subjects during submaximal exercise. Chest 1999; 116: 1208-17.

14. Sharman JE, Stowasser M. Australian association for exercise and sports science position statement on exercise and hypertension. J Sci Med Sport 2009; 12: 252-7.

15. ATS Committee on Proficiency Standards for Clinical Pulmonary Function Laboratories. ATS statement: 
guidelines for the six-minute walk test. Am J Respir Crit Care Med 2002; 166: 111-7.

16. Dangour AD, Albala C, Allen E, Grundy E, Walker DG, Aedo S, et al. Effect of nutrition supplement and physical activity program on pneumonia and walking capacity in Chilean people: a factorial cluster randomized trial. Plos Med 2011; 8.

17. Beck At, Ward Ch, Mendelson M, Mock J, Erbaugh J. An inventory for measuring depression. Arch Gen Psychiatry 1961; 4: 561-71.

18. Ridley J, Hoey K, Ballagh-Howes N. The exerciseduring-hemodialysis program: report on a pilot study. CANNT J 1999; 9(3): 20-6.
19. Painter P, Carlson L, Carey S, Paul SM, Myll J. Physical functioning and health-related quality-of-life changes with exercise training in hemodialysis patients. Am J Kidney Dis 2000; 35: 482-92.

20. Parsons TL, Toffelmire EB, King-VanVlack CE. The effect of an exercise program during hemodialysis on dialysis efficacy, blood pressure and quality of life in end-stage renal disease (ESRD) patients. Clin Nephrol 2004; 61: 261-74.

21. Oh-Park M, Fast A, Gopal S, Lynn R, Frei G, Drenth R, Zohman L. Exercise for the dialyzed: aerobic and strength training during hemodialysis. Am J Phys Med Rehabil 2002; 81: 814-21. 